This item was submitted to Loughborough's Research Repository by the author.

Items in Figshare are protected by copyright, with all rights reserved, unless otherwise indicated.

\title{
Hybrid equilibrium finite element formulation for composite beams with partial
} interaction

PLEASE CITE THE PUBLISHED VERSION

http://dx.doi.org/10.1016/j.compstruct.2013.09.062

PUBLISHER

(C) Elsevier

VERSION

AM (Accepted Manuscript)

\section{PUBLISHER STATEMENT}

This work is made available according to the conditions of the Creative Commons Attribution-NonCommercialNoDerivatives 4.0 International (CC BY-NC-ND 4.0) licence. Full details of this licence are available at: https://creativecommons.org/licenses/by-nc-nd/4.0/

\section{LICENCE}

CC BY-NC-ND 4.0

\section{REPOSITORY RECORD}

Santos, Hugo A.F.A., and Vadim V. Silberschmidt. 2019. "Hybrid Equilibrium Finite Element Formulation for Composite Beams with Partial Interaction”. figshare. https://hdl.handle.net/2134/25636. 


\title{
Hybrid equilibrium finite element formulation for composite beams with partial interaction
}

\author{
H.A.F.A. Santos *, V.V. Silberschmidt \\ Wolfson School of Mechanical and Manufacturing Engineering, Loughborough University, Loughborough, Leicestershire LE11 3TU, UK
}

\begin{abstract}
A B S T R A C T
Thanks to their various benefits, composite beams have been increasingly used in various applications. This study will focus on two-layer composite beams with a flexible shear interface between layers. The finite element method, in particular its displacement-based formulation, has been recognized as the most popular method for numerical analysis of composite beams. However, when applied to Timoshenko beams with partial interaction, the displacement-based formulation may suffer from the so-called shear-locking and slip-locking phenomena, leading to erroneous solutions. Hybrid and mixed finite element formulations have been viewed as competitive alternatives, since they naturally avoid locking effects. Special types of these formulations are the so-called equilibrium-based formulations, producing statically admissible solutions. This work introduces for the first time an equilibrium-based finite element formulation for the analysis of Timoshenko composite beams with partial interaction. The formulation relies on a variational principle of complementary energy involving only force/moment-like variables as fundamental unknown fields. The approximate field variables are selected such that all equilibrium equations hold in strong form. The inter-element equilibrium is enforced by resorting to the Lagrangian multiplier method. Unlike traditional displacement-based finite element formulations, the proposed scheme is naturally free from both shearand slip-locking phenomena. The accuracy and effectiveness of the new formulation is numerically assessed through the analysis of several numerical examples. In particular, the ability of the formulation to model accurately both very flexible and very stiff shear connections is numerically shown.
\end{abstract}

Keywords:

Composite beams

Timoshenko theory

Partial interaction

Complementary energy

Finite elements

Hybrid method

Equilibrium

\section{Introduction}

Composite materials have a number of advantages over their isotropic counterparts, including low density, high strength and stiffness, as well as tailoring their properties by changing fibre orientation. Composite beams have been increasingly used in various industries, such as aerospace, automotive, nuclear, marine, biomedical, and civil engineering. Composite sandwich and laminated beams are typical components used in aerospace structures, while in civil engineering, steel-concrete composite beams in buildings and bridges, wood-concrete floor systems, and concrete beams externally reinforced with laminates are often encountered. This study focuses on two-layer composite beams with a single flexible shear interface, such as steel-concrete beams or two-layer wooden beams.

The mechanical behaviour of composite structures depends to a large extent on the character of bonding. If the layers are connected continuously by means of strong adhesives, the mechanical assumption of a perfect bond between the layers is reasonable. However, the layers are often connected non-continuously, by means of

\footnotetext{
* Corresponding author.

E-mail address: hugofreixialsantos@gmail.com (H.A.F.A. Santos).
}

connectors, such as shear studs and nails, which are not rigid. Therefore, some slip and uplift can occur at the interlayer. While the uplift is often small and can be neglected, the interlayer slip significantly affects the behaviour of composite elements. This phenomenon is called partial (or incomplete) interaction and is an important issue in composite structures [24]. In fact, the inclusion of the interlayer-slip effect in the theory of composite beams is essential for optimal design and accurate representation in simulations of the actual mechanical behaviour of composite structures with partial interaction. Many efforts and a large number of research studies have been devoted to obtain the solution to this problem.

The first one-dimensional composite beam model with flexible shear connectors was developed by Newmark et al. [23], in which two layers were assumed to be connected in such a way that vertical separation did not occur between the components. Both layers were assumed to follow the kinematic assumptions of the EulerBernoulli beam theory. Fairly recently, analytical solution methods based on Newmark's model for the static response of two-layer beams with interlayer slip in the linear-elastic regime were proposed, e.g., in $[39,15,19,28,27,16]$. All these studies are based on the Euler-Bernoulli beam theory, which considers that planes that are perpendicular to the beam axis before bending will remain 
plane and perpendicular to it after deformation. In other words, the Euler-Bernoulli beam theory does not consider any effect of transverse shear deformation. However, for beams either with a small span-to-depth ratio, low shear rigidity, or continuous spans, the effect of transverse shear deformation is not small and, therefore, cannot be disregarded. The shear deformation effect was incorporated in the analysis for the first time by Murakami [21], who used Timoshenko's (also often referred to as first-order) beam theory to represent the deformation of the beam layers. Analytical solutions for two-layer beam elements based on the first-order shear deformation theory, in which different shear deformations were allowed in the two layers, were derived in [36]. A formulation for the analysis of members with non-uniformly distributed shear connectors and interlayer transverse separation was proposed in [6]. For a review on various Euler-Bernoulli and Timoshenko-based models that were proposed in the literature for the analysis of composite beams with partial interaction the reader is referred to [20].

Composite elements based on (more sophisticated) higher-order shear deformation beam theories, which incorporate the warping of the beam section produced by shear deformation by taking a nonlinear variation of the axial displacement of the fibres over the beam depth, were proposed in, e.g., [43,11,1,38,22,5]. Numerical methods, in particular the finite element method, have also been widely used in the analysis of both linear and non-linear composite beams with partial interaction. Displacement-based Timoshenko finite element formulations for the analysis of partial interaction composite members were developed in, e.g., $[2,10,7]$. However, these formulations may suffer from the shear-locking and slip-locking phenomena.

As is well known, classical displacement-based Timoshenko beam elements with the same interpolation of both the transverse and rotation fields behave very stiff in the thin-beam limit, i.e., as the length-to-thickness ratio becomes large. Such a behaviour is known as shear-locking and is due to the inconsistency of the interpolation for the transverse and rotation fields. To overcome shear-locking, one may use equal interpolation for both fields, but use a lower-order polynomial for the shear strain. This is often realized by using selective integration, in which reduced-order integration is used to evaluate the stiffness coefficients associated with the transverse shear strain, and all other coefficients of the stiffness matrix are evaluated using full integration. Unfortunately, this procedure leads to spurious energy modes [26]. Alternative approaches based on hybrid and mixed finite element formulations to alleviate shear-locking were pursued by many investigators, e.g., $[18,25,37,17]$. While the latter are based on multi-field variational principles, such as a two-field Hellinger-Reissner principle or a three-field Hu-Washizu principle, the former rely on modified variational principles with relaxed continuity requirements across element boundaries, thus assuming independent approximations for field variables within the element and along the boundaries.

As for the slip-locking phenomenon, it occurs due to the coupling between the transverse and axial displacement fields and it can lead to erroneous oscillations in the slip field and a considerable reduction of the optimal rate of convergence for high values of the connection stiffness $[7,8]$. Models that attempt to overcome these limitations within the framework of composite members with partial interaction were proposed in $[30,4,9,3,36]$. Alternative strategies to alleviate the slip-locking behaviour in the classical displacement-based finite element formulation were recently adopted in [14], where techniques based on the assumed strain method, discrete strain gap method, and kinematic interpolatory method were introduced.

Hybrid and mixed finite element formulations can be used to naturally avoid locking effects, without a need to resort to any numerical tricks. Special types of these formulations are the socalled equilibrium-based formulations, first introduced in [12,13] for small elastic deformation problems. They are very often derived from complementary variational principles. In these formulations, the approximate fields are chosen so that the stress fields are in equilibrium or, in other words, internal equilibrium and continuous stress transmission between elements are satisfied exactly. In contrast, the compatibility differential equations and the Dirichlet boundary conditions are only satisfied in a weak form. The numerical solutions obtained with these models are called statically admissible solutions. These formulations have a special appeal for practical design engineers, despite the popularity of the conventional displacement formulations, due to the exact transmission of stresses across boundaries between adjacent structural members. This avoids the need for the 'averaging' procedures required to obtain unique nodal values of stresses when resorting to displacement formulations. In fact, in structural engineering design, the stresses are often the variables of most interest, whereas the displacements are of secondary interest. Indeed, although displacement formulations can lead to sufficiently accurate displacement fields, the corresponding stress fields may be highly erroneous. This occurs since the accuracy of the approximate displacement field rapidly deteriorates when differentiations are required to compute other results, such as stresses or strains. In contrast, for equilibrium formulations, the stresses are computed as fundamental unknowns. Examples of equilibrium-based finite element formulations for geometrically non-linear beam problems were presented in $[33,35,32]$. An equilibrium-based formulation for non-linear elastic cables can be found in [34]. For further details on these formulations the reader is referred to [31].

A finite element formulation for non-linear composite beams with partial interaction considering only forces and moments as fundamental unknowns that satisfy a priori the equilibrium differential equations was proposed in $[30,29]$. However, besides being based on the Euler-Bernoulli beam theory, disregarding therefore shear deformation effects, such a formulation leads to slip distributions that are not continuous across the inter-element boundaries. A mixed formulation also for non-linear Euler-Bernoulli-based composite beams was introduced in [4]. Such a formulation not only uses two types of approximations, i.e., displacements and internal forces/moments, which require to comply with additional stability conditions, it also leads to solutions that do not satisfy the equilibrium differential equations of the problem in a strong form.

In this work, a hybrid equilibrium-based finite element formulation for the analysis of composite Timoshenko beams with partial interaction is introduced for the first time. This formulation relies on a variational principle of complementary energy only involving force- and moment-like variables as fundamental unknown fields, and leads to statically admissible solutions, i.e., solutions that satisfy all the equilibrium conditions in a strong form. Unlike traditional displacement-based finite element formulations, the proposed scheme is naturally free from both shear- and slip-locking phenomena. Feasibility and effectiveness of the proposed formulation is numerically demonstrated through the analysis of several numerical tests.

\section{Boundary-value problem}

The aim of this study is to investigate the behaviour of composite beams with two material layers with a shear flexible interface as shown in Fig. 1. As discussed in the previous section, the Timoshenko (or first-order shear deformation) theory is adopted to describe the deformation of the beam layers. Hence, transverse shear deformations are allowed, and the rotation angle and shear deformations are assumed to be identical in the two layers. In addition, the following assumptions are considered: (i) no uplift occurs between the two layers (i.e., both layers have the same transverse displacement); (ii) slip can occur at the interlayer (i.e., partial interaction is assumed); (iii) the layers are connected continuously 


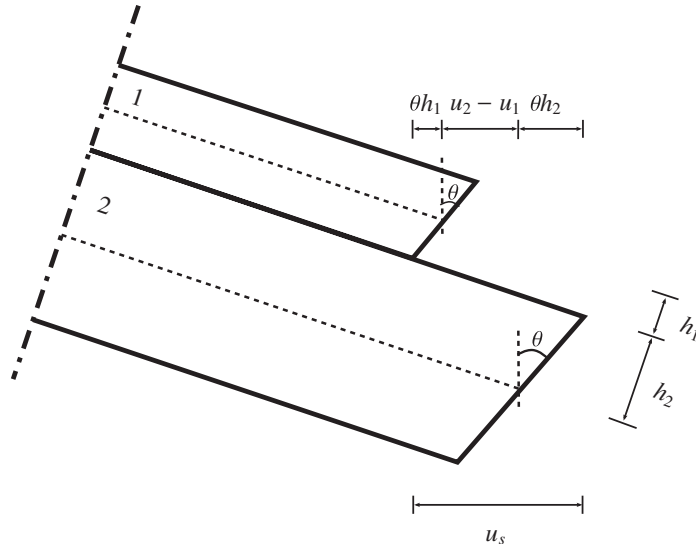

Fig. 1. Beam's kinematics.

by means of longitudinally distributed shear connectors; and (iv) the material behaviour is linear elastic.

An initially straight, planar, two-layer Timoshenko beam whose centroidal axis is parameterized by $x \in[0, L]$, with $L$ the length of the beam, is considered. The centroidal axis is decomposed into an internal part, represented by $\Omega=] 0, L[$, and a boundary part, identified by $\Gamma=\Gamma_{N} \cup \Gamma_{D}=\{0, L\}$, where $\Gamma_{N}$ and $\Gamma_{D}$ correspond to the Neumann and Dirichlet boundaries, respectively, such that $\Gamma_{N} \cap \Gamma_{D}=\emptyset$.

The beam is subjected to a distributed transverse load $q(x)$ and a bending moment $m(x)$ applied in $\Omega$; a concentrated axial force $\bar{N}$, a shear force $\bar{V}$ and a bending moment $\bar{M}$ applied on $\Gamma_{N}$; and a prescribed axial displacement $\bar{u}$, a transverse displacement $\bar{w}$ and a prescribed rotation $\bar{\theta}$ defined on $\Gamma_{D}$. The loads are assumed to act at the centroidal axis of the beam.

$V$ and $Q_{s}$ are the shear force and interlayer slip flux fields of the beam, respectively. $N_{i}$ and $M_{i}$ denote the axial force and bending moment fields of layer $i$. The layers are denoted by indices 1 and 2 ; see Fig. $1 . h_{i}$ corresponds to the distance from the centroidal axis of layer $i$ to the interface. $u_{i}$ denotes the centroidal axial displacement of layer $i$. The rotation angle of the cross-section of the layers is represented by $\theta . w$ is the centroidal transverse displacement of the beam.

Let us consider the equilibrium of the infinitesimal beam element of length $d x$ depicted in Fig. 2. It can be easily seen that the equilibrium of such an element can be expressed by the following differential equations in $\Omega$

$V^{\prime}+q(x)=0$

$M_{1}^{\prime}+M_{2}^{\prime}+Q_{s} h-V-m(x)=0$,

$Q_{s}+N_{1}^{\prime}=0$,

$Q_{s}-N_{2}^{\prime}=0$, representing equilibrium of shear forces, bending moments and axial forces, respectively, where $(\cdot)^{\prime}$ stands for the derivative of $(\cdot)$ with respect to $x$.

The equilibrium conditions defined at the cross-section can be expressed as

$N=N_{1}+N_{2}$

$M=M_{1}+M_{2}-N_{1} h$

with $h=h_{1}+h_{2}$. $N$ and $M$ stand for the axial force and bending moment fields.

The compatibility differential equations, according to the Timoshenko beam theory, are given in $\Omega$ by

$$
\begin{aligned}
& \gamma=w^{\prime}-\theta, \\
& \kappa=-\theta^{\prime}, \\
& \varepsilon_{1}=u_{1}^{\prime}, \\
& \varepsilon_{2}=u_{2}^{\prime},
\end{aligned}
$$

with $\gamma$ being the shear deformation, $\kappa$ the bending curvature and $\varepsilon$ $(i=1,2)$ the axial deformation of layer $i$.

The cross-sectional compatibility relationship defines the interface slip field $u_{s}$ as

$u_{s}=u_{2}-u_{1}+\theta h$, in $\Omega$.

The constitutive relationships in $\Omega$ are taken as

$V=C \gamma$,

$M_{1}=-E_{1} I_{1} \theta^{\prime}$,

$M_{2}=-E_{2} I_{2} \theta^{\prime}$

$N_{1}=E_{1} A_{1} \varepsilon_{1}$

$N_{2}=E_{2} A_{2} \varepsilon_{1}$,

$Q_{s}=k_{s} u_{s}$

with $C=k_{1} G_{1} A_{1}+k_{2} G_{2} A_{2}$ being the shear rigidity of the whole cross-section, where $k_{i}$ is the shear correction coefficient of layer $i$, which depends on the cross-section geometry of the layer. $E_{i}, G_{i}, I_{i}$ and $A_{i}$ denote the Young's modulus, shear modulus, moment of inertia and cross-sectional area, respectively, of layer $i . k_{s}$ stands for the interlayer slip modulus.

The Dirichlet (or compatibility) boundary conditions of the problem are given on $\Gamma_{D}$ as follows

$u_{1}-\bar{u}_{1}=0$,

$u_{2}-\bar{u}_{2}=0$,

$w-\bar{w}=0$,

$\theta-\bar{\theta}=0$.

The Neumann (or equilibrium) boundary conditions of the problem are given on $\Gamma_{N}$ as follows

$n N_{1}-\bar{N}_{1}=0$,

$n N_{2}-\bar{N}_{2}=0$,

$n V-\bar{V}=0$,

$\bar{M}-n\left(M_{1}+M_{2}\right)=0$,

with

$n= \begin{cases}1 & \text { if } x=L \\ -1 & \text { if } x=0\end{cases}$

\section{Variational setting}

Let us introduce the total potential energy functional $\Pi_{p}: \mathcal{U}_{k}(\Omega) \rightarrow \mathcal{R}$ given by

$\Pi_{p}\left(w, u_{1}, u_{2}, u_{s}, \theta\right)=\frac{1}{2} \int_{\Omega}\left(D \theta^{\prime 2}+E_{1} A_{1} u_{1}^{\prime 2}+E_{2} A_{2} u_{2}^{\prime 2}+C\left(w^{\prime}-\theta\right)^{2}\right.$

$\left.+k_{s} u_{s}^{2}\right) d \Omega-\int_{\Omega}(q w+m \theta) d \Omega-\left[\bar{N}_{1} u_{1}\right]_{\Gamma_{N}}-\left[\bar{N}_{2} u_{2}\right]_{\Gamma_{N}}-[\bar{V} w]_{\Gamma_{N}}+[\bar{M} \theta]_{\Gamma_{N}}$

Fig. 2. Equilibrium of infinitesimal beam element. 
with $D=E_{1} I_{1}+E_{2} I_{2}$. $\mathcal{U}_{k}$ represents the kinematically admissible space defined as

$$
\begin{aligned}
\mathcal{U}_{k} & =\left\{\left(w, u_{1}, u_{2}, u_{s}, \theta\right) \in \mathcal{H}^{1} \times \mathcal{H}^{1} \times \mathcal{H}^{1} \times \mathcal{H}^{0} \times \mathcal{H}^{1} \mid u_{s}\right. \\
& =u_{2}-u_{1}+\theta h \text { in } \Omega, u_{1}-\bar{u}_{1}=0, u_{2}-\bar{u}_{2}=0 \\
& \left.w-\bar{w}=0, \theta-\bar{\theta}=0 \text { on } \Gamma_{D}\right\} .
\end{aligned}
$$

It can be shown that, under the subsidiary conditions (3), the total potential energy renders a stationary principle. To see this, let us consider the first variation of $\Pi_{p}$ with respect to its arguments, which reads

$$
\begin{aligned}
\delta \Pi_{p}= & \int_{\Omega}\left(D \theta^{\prime} \delta \theta^{\prime}+E_{1} A_{1} u_{1}^{\prime} \delta u_{1}^{\prime}+E_{2} A_{2} u_{2}^{\prime} \delta u_{2}^{\prime}+C\left(w^{\prime}-\theta\right)\left(\delta w^{\prime}-\delta \theta\right)\right. \\
& \left.+k_{s} u_{s} \delta u_{s}\right) d \Omega-\int_{\Omega}(q \delta w+m \delta \theta) d \Omega-\left[\bar{N}_{1} \delta u_{1}\right]_{\Gamma_{N}}-\left[\bar{N}_{2} \delta u_{2}\right]_{\Gamma_{N}} \\
& -[\bar{V} \delta w]_{\Gamma_{N}}+[\bar{M} \delta \theta]_{\Gamma_{N}} .
\end{aligned}
$$

This, upon substitution of the constitutive relations (5) and the compatibility Eq. (4), and after integrating by parts, gives rise to

$$
\begin{aligned}
\delta \Pi_{p}= & \int_{\Omega}\left(\left(M_{1}^{\prime}+M_{2}^{\prime}-V+Q_{s} h-m\right) \delta \theta-\left(V^{\prime}+q\right) \delta w\right. \\
& \left.-\left(N_{1}^{\prime}+Q_{s}\right) \delta u_{1}+\left(Q_{s}-N_{2}^{\prime}\right) \delta u_{2}\right) d \Omega+\left[\left(N_{1}-\bar{N}_{1}\right) \delta u_{1}\right]_{\Gamma_{N}} \\
& +\left[\left(N_{2}-\bar{N}_{2}\right) \delta u_{2}\right]_{\Gamma_{N}}+[(V-\bar{V}) \delta w]_{\Gamma_{N}}+\left[\left(\bar{M}-M_{1}-M_{2}\right) \delta \theta\right]_{\Gamma_{N}} .
\end{aligned}
$$

It can now be easily seen that the stationarity conditions of the total potential energy, which are determined from $\delta \Pi_{p}=0$, are the equilibrium conditions (1) and (7). Standard displacement-based finite element formulations are typically constructed on the basis of the principle of stationary total potential energy.

Following [32,40], the total potential energy functional can be transformed into the total complementary energy functional $\Pi_{c}: \mathcal{U}_{s}(\Omega) \rightarrow \mathcal{R}$ defined as

$\Pi_{c}\left(M_{1}, M_{2}, N_{1}, N_{2}, V, Q_{s}\right)=\frac{1}{2} \int_{\Omega} \frac{M_{1}^{2}}{E_{1} I_{1}}+\frac{M_{2}^{2}}{E_{2} I_{2}}+\frac{N_{1}^{2}}{E_{1} A_{1}}+\frac{N_{2}^{2}}{E_{2} A_{2}}+\frac{V^{2}}{C}$

$\left.+\frac{Q_{s}^{2}}{k_{s}}\right) d \Omega-\left[N_{1} \bar{u}_{1}\right]_{\Gamma_{D}}-\left[N_{2} \bar{u}_{2}\right]_{\Gamma_{D}}-[V \bar{w}]_{\Gamma_{D}}-\left[\left(M_{1}+M_{2}\right) \bar{\theta}\right]_{\Gamma_{D}}$

where $\mathcal{U}_{s}$ represents the statically admissible space defined as

$$
\begin{aligned}
& \mathcal{U}_{s}=\left\{\left(M_{1}, M_{2}, N_{1}, N_{2}, V, Q_{s}\right)\right. \\
& \quad \in \mathcal{H}^{0} \times \mathcal{H}^{0} \times \mathcal{H}^{0} \times \mathcal{H}^{0} \times \mathcal{H}^{0} \times \mathcal{H}^{0} \\
& \quad \mid V^{\prime}+q(x)=0, M_{1}^{\prime}+M_{2}^{\prime}+Q_{s} h-V-m=0, \\
& Q_{s}+N_{1}^{\prime}=0, Q_{s}-N_{2}^{\prime}=0 \text { in } \Omega \text { and } n N_{1}-\bar{N}_{1}=0, \\
& \left.n N_{2}-\bar{N}_{2}=0, n V-\bar{V}=0, \bar{M}-n\left(M_{1}+M_{2}\right)=0 \text { on } \Gamma_{N}\right\} .
\end{aligned}
$$

Under the subsidiary conditions of equilibrium (1) and (7), the total complementary energy renders a stationary principle. To see this, let us consider the first variation of $\Pi_{c}$ under the subsidiary conditions of equilibrium, which gives

$$
\begin{aligned}
\delta \Pi_{c} & =\int_{\Omega}\left(\frac{M_{1}}{E_{1} I_{1}} \delta M_{1}+\frac{M_{2}}{E_{2} I_{2}} \delta M_{2}+\frac{N_{1}}{E_{1} A_{1}} \delta N_{1}+\frac{N_{2}}{E_{2} A_{2}} \delta N_{2}+\frac{V}{C} \delta V\right. \\
& \left.+\frac{Q_{s}}{k_{S}} \delta Q_{s}\right) d \Omega-\int_{\Omega}\left(\left(\delta M_{1}^{\prime}+\delta M_{2}^{\prime}+\delta Q_{s} h-\delta V\right) \theta-\delta V^{\prime} w-\left(\delta N_{1}^{\prime}+\delta Q_{s}\right) u_{1}\right. \\
& \left.+\left(\delta Q_{s}-\delta N_{2}^{\prime}\right) u_{2}\right) d \Omega-\left[\delta N_{1} \bar{u}_{1}\right]_{\Gamma_{D}}-\left[\delta N_{2} \bar{u}_{2}\right]_{\Gamma_{D}}-[\delta V \bar{w}]_{\Gamma_{D}} \\
& -\left[\left(\delta M_{1}+\delta M_{2}\right) \bar{\theta}\right]_{\Gamma_{D}}-\left[\delta N_{1} u_{1}\right]_{\Gamma_{N}}-\left[\delta N_{2} u_{2}\right]_{\Gamma_{N}}-[\delta V w]_{\Gamma_{N}} \\
& +\left[\left(\delta M_{1}+\delta M_{2}\right) \theta\right]_{\Gamma_{N}} .
\end{aligned}
$$

This, upon substitution of the constitutive relations and after integrating by parts, gives rise to

$$
\begin{aligned}
\delta \Pi_{c}= & \int_{\Omega}\left(\left(\kappa+\theta^{\prime}\right) \delta M_{1}+\left(\kappa+\theta^{\prime}\right) \delta M_{2}+\left(\gamma-w^{\prime}+\theta\right) \delta V\right. \\
& \left.+\left(\varepsilon_{1}-u_{1}^{\prime}\right) \delta N_{1}+\left(\varepsilon_{2}-u_{2}^{\prime}\right) \delta N_{2}+\left(u_{s}-u_{2}+u_{1}-\theta h\right) \delta Q_{s}\right) d \Omega \\
& +\left[\left(u_{1}-\bar{u}_{1}\right) \delta N_{1}\right]_{\Gamma_{D}}+\left[\left(u_{2}-\bar{u}_{2}\right) \delta N_{2}\right]_{\Gamma_{D}}+[(w-\bar{w}) \delta V]_{\Gamma_{D}} \\
& +\left[(\theta-\bar{\theta})\left(\delta M_{1}+\delta M_{2}\right)\right]_{\Gamma_{D}} .
\end{aligned}
$$

This clearly shows that the total complementary energy renders a stationarity principle under the compatibility conditions (3), (4) and (6). Hence, force-based finite element formulations can be constructed on the basis of this principle.

Let us now assume that the entire domain $\Omega$ is partitioned in subdomains $\Omega_{e} \subset \Omega$, such that $\Omega=\cup_{e=1}^{n_{e}} \Omega_{e}$ in which $n_{e}$ represents the number of beam elements. If the inter-element equilibrium conditions and Neumann boundary conditions are relaxed within the framework of the complementary energy principle, then, the following augmented Lagrangian, or hybrid complementary energy, $L_{c}: \mathcal{U}_{s}^{*}(\Omega) \rightarrow \mathcal{R}$ must be considered

$$
\begin{aligned}
L_{c}= & \sum_{e=1}^{n_{e}} \Pi_{c, e}+\sum_{i=1}^{n_{\text {int }}}\left(\lambda_{\Gamma_{i}}^{N_{1}} \llbracket N_{1} \rrbracket_{\Gamma_{i}}+\lambda_{\Gamma_{i}}^{N_{2}} \llbracket N_{2} \rrbracket_{\Gamma_{i}}+\lambda_{i}^{V} \llbracket V \rrbracket_{\Gamma_{i}}+\lambda_{i}^{M_{1}} \llbracket M_{1} \rrbracket_{\Gamma_{i}}\right. \\
& \left.+\lambda_{i}^{M_{2}} \llbracket M_{2} \rrbracket_{\Gamma_{i}}+\lambda_{i}^{Q_{s}} \llbracket Q_{s} \rrbracket_{\Gamma_{i}}\right),
\end{aligned}
$$

where $n_{\text {int }}$ is the number of inter-element and Neumann boundaries and $\Gamma_{i}$ is the inter-element boundary $i$. $\llbracket(\cdot) \rrbracket$ stands for the jump of (.) on $\Gamma_{i}$. $\mathcal{U}_{s}^{*}$ represents a modified statically admissible space defined as

$\mathcal{U}_{s}^{*}=\left\{\left(M_{1}, M_{2}, N_{1}, N_{2}, V, Q_{s}\right) \in \mathcal{H}^{0} \times \mathcal{H}^{0} \times \mathcal{H}^{0} \times \mathcal{H}^{0} \times \mathcal{H}^{0} \times \mathcal{H}^{0}\right.$

$\mid V^{\prime}+q(x)=0, M_{1}^{\prime}+M_{2}^{\prime}+Q_{s} h-V-m=0$,

$$
\left.Q_{s}+N_{1}^{\prime}=0, Q_{s}-N_{2}^{\prime}=0 \text { in } \Omega\right\}
$$

and $\lambda_{i}^{N_{1}}, \lambda_{i}^{N_{2}}, \lambda_{i}^{V}, \lambda_{i}^{M_{1}}, \lambda_{i}^{M_{1}}$ and $\lambda_{i}^{Q_{s}}$ are appropriate energy-conjugate Lagrange multipliers.

The stationarity conditions of the hybrid complementary energy $L_{c}$, obtained from $\delta L_{c}=0$, are Eqs. (3), (6), (7) and, in addition, the inter-element equilibrium conditions on $\Gamma_{\text {int }}$

$\llbracket N_{1} \rrbracket=0$,

$\llbracket N_{2} \rrbracket=0$,

$\llbracket V \rrbracket=0$,

$\llbracket M_{1} \rrbracket=0$,

$\llbracket M_{2} \rrbracket=0$,

$\llbracket Q_{s} \rrbracket=0$,

and inter-element compatibility conditions on $\Gamma_{\text {int }}$

$\lambda^{N_{1}}-u_{1}=0$

$\lambda^{N_{2}}-u_{2}=0$,

$\lambda^{V}-w=0$

$\lambda^{M_{1}}-\theta=0$,

$\lambda^{M_{2}}-\theta=0$,

$\lambda^{Q_{s}}-u_{s}=0$,

with $\Gamma_{\text {int }}=\cup_{i=1}^{n_{\text {int }}} \Gamma_{i}$.

\section{Finite element approximations}

A hybrid equilibrium finite element formulation for the analysis of Timoshenko composite beams with partial interaction is introduced in this section. This formulation relies on the hybrid form of the complementary energy principle presented in the preceding section.

Let us beforehand define $\mathcal{H}_{h}^{0}$ and $\mathcal{H}_{h}^{1}$ as families of closed finitedimensional subspaces of $\mathcal{H}^{0}$ and $\mathcal{H}^{1}$, respectively. A finite element 
approximation of (14) consists of seeking $\left(M_{1}^{h}, M_{2}^{h}, N_{1}^{h}, N_{2}^{h}, V^{h}, Q^{h}\right) \in \mathcal{U}_{s}^{* h}$ such that the condition $\delta L_{c}=0$ holds for all $\left(\delta M_{1}^{h}, \delta M_{2}^{h}, \delta N_{1}^{h}, \delta N_{2}^{h}, \delta V^{h}, \delta Q^{h}\right) \in \mathcal{V}_{s}^{* h}$, where $\mathcal{U}_{s}^{* h} \subset \mathcal{U}_{s}^{*}$ and $\mathcal{V}_{s}^{* h} \subset \mathcal{V}_{s}$ represent the discrete forms of the modified statically admissible spaces.

For the sake of simplicity, and without loss of generality, let us consider the case of beams under transverse uniformly distributed loads and zero-valued distributed bending moments, i.e., $q=$ cte and $m=0$. Let us also consider homogeneous Dirichlet boundary conditions.

The simplest finite-dimensional approximation to $\mathcal{U}_{s}^{* h} \subset \mathcal{U}_{s}^{*}$ is obtained assuming the following trial finite element approximations for bending moments

$M_{1}^{h}=M_{1}^{i}\left(1-\frac{x}{L}\right)+M_{1}^{j} \frac{x}{L}-4 M_{1}^{k} \frac{x(L-x)}{L^{2}}$,

$M_{2}^{h}=M_{2}^{i}\left(1-\frac{x}{L}\right)+M_{2}^{j} \frac{x}{L}-4 M_{2}^{k} \frac{x(L-x)}{L^{2}}$,

where the pairs $M_{1}^{i}, M_{2}^{i}$ and $M_{1}^{j}, M_{2}^{j}$ are the bending moments of beams 1 and 2 defined at $x=0$ and $x=L$, respectively. $M_{1}^{k}$ and $M_{2}^{k}$ are the mid-span bending moments of beams 1 and 2 .

The approximations for shear and axial forces are taken as

$V^{h}=V_{0}-q x$

$N_{1}^{h}=N_{10}+n_{1} \frac{x}{h L}+n_{2} \frac{x^{2}}{h L^{2}}$,

$N_{2}^{h}=N_{20}+n_{1} \frac{x}{h L}+n_{2} \frac{x^{2}}{h L^{2}}$,

with

$n_{1}=M_{1}^{j}-M_{1}^{i}+M_{2}^{j}-M_{2}^{i}+4\left(M_{1}^{k}+M_{2}^{k}\right)-V_{0} L$,

$n_{2}=-4\left(M_{1}^{k}+M_{2}^{k}\right)+\frac{q L^{2}}{2}$

Here, $V_{0}, N_{10}$ and $N_{20}$ represent the shear force and axial force parameters defined at $x=0$.

The approximation for shear flux is assumed as

$Q_{s}^{h}=n_{1} \frac{1}{h L}+2 n_{2} \frac{x}{h L^{2}}$.

A Galerkin approach is adopted, i.e., the problem is numerically approached assuming the same trial and test approximation function spaces.

It is worth noting that these approximations are such that all equilibrium differential equations hold in a strong form. It is also important to remark that the approximations for bending moments are selected so that the inter-element moment equilibrium is satisfied a priori. This avoids the need to enforce inter-element continuity of moments through the augmented Lagrangian $L_{c}$.

The discrete form of the hybrid complementary energy is therefore obtained as

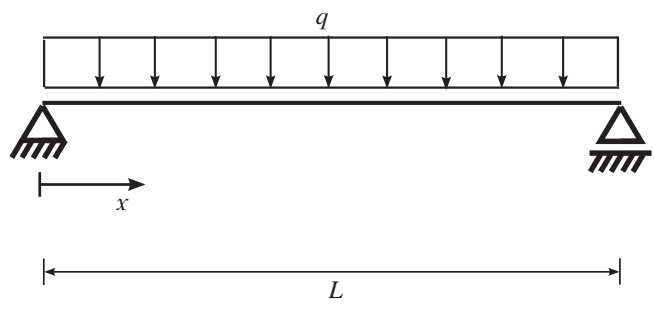

Fig. 4. Simply-supported beam.

Table 1

Simply-supported beam problem with $k_{s}=0.243 \mathrm{kN} / \mathrm{cm}^{2}$ : mid-span transverse displacement $w^{h}(L / 2)$, interlayer slip $u_{s}^{h}(0)$ and rotation $\theta^{h}(0)$.

\begin{tabular}{llll}
\hline$n_{e}$ & $w^{h}(L / 2)(\mathrm{cm})$ & $u_{s}^{h}(0)(\mathrm{cm})$ & $\theta^{h}(0)(\mathrm{rad})$ \\
\hline 2 & 0.27008867 & -0.09185267 & 0.00307252 \\
4 & 0.27005427 & -0.08078438 & 0.00307252 \\
8 & 0.27005307 & -0.07767260 & 0.00307252 \\
16 & 0.27005301 & -0.07683492 & 0.00307252 \\
32 & 0.27005300 & -0.07661785 & 0.00307252 \\
64 & 0.27005300 & -0.07656262 & 0.00307252 \\
128 & 0.27005300 & -0.07654870 & 0.00307252 \\
Exact & 0.27005300 & -0.07654403 & 0.00307252 \\
\hline
\end{tabular}

Table 2

Simply-supported beam problem with $k_{s}=0.243 \mathrm{kN} / \mathrm{cm}^{2}$ : mid-span bending moment $M^{h}(L / 2)$, shear force $V^{h}(0)$, and interlayer shear flux $Q_{s}^{h}(0)$.

\begin{tabular}{rlll}
\hline$n_{e}$ & $M^{h}(L / 2)(\mathrm{kN} \mathrm{cm})$ & $V_{s}^{h}(0)(\mathrm{kN})$ & $Q_{s}^{h}(0)(\mathrm{kN} / \mathrm{cm})$ \\
\hline 2 & 3906.25 & 62.5 & -0.02232020 \\
4 & 3906.25 & 62.5 & -0.01963060 \\
8 & 3906.25 & 62.5 & -0.01887444 \\
16 & 3906.25 & 62.5 & -0.01867088 \\
32 & 3906.25 & 62.5 & -0.01861814 \\
64 & 3906.25 & 62.5 & -0.01860472 \\
128 & 3906.25 & 62.5 & -0.01860133 \\
Exact & 3906.25 & 62.5 & -0.01860020 \\
\hline
\end{tabular}

$L_{c}^{h}=\sum_{e=1}^{n_{e}} \Pi_{c, e}^{h}+\sum_{i=1}^{n_{\text {int }}}\left(\lambda_{i}^{N_{1}} \llbracket N_{1}^{h} \rrbracket_{i}+\lambda_{i}^{N_{2}} \llbracket N_{2}^{h} \rrbracket_{i}+\lambda_{i}^{V} \llbracket V^{h} \rrbracket_{i}+\lambda_{i}^{Q_{s}} \llbracket Q_{s}^{h} \rrbracket_{i}\right)$,

where $n_{e}$ is the number of beam elements and $n_{\text {int }}$ is the number of inter-element and Neumann boundaries. Note that the term corresponding to the enforcement of the inter-element moment equilibrium conditions was dropped, as it is not necessary in light of the arguments mentioned above.

Considering a single finite element, differentiation of $L_{c}^{h}$ with respect to the unknown element parameters gives the governing system of equations as

$$
\left[\begin{array}{cc}
\boldsymbol{F}_{e} & \boldsymbol{A}_{e}^{T} \\
\boldsymbol{A}_{e} & \boldsymbol{0}
\end{array}\right]\left[\begin{array}{c}
\boldsymbol{s}_{e} \\
\boldsymbol{\lambda}
\end{array}\right]=\left[\begin{array}{l}
\mathbf{0} \\
\mathbf{0}
\end{array}\right],
$$

where $\boldsymbol{s}_{e}$ is the vector of element parameters collected as

$\boldsymbol{s}_{e}=\left[\begin{array}{lllllllll}M_{1}^{i} & M_{1}^{j} & M_{1}^{k} & M_{2}^{i} & M_{2}^{j} & M_{2}^{k} & N_{10} & N_{20} & V_{0}\end{array}\right]^{T}$

and $\lambda$ is the vector of Lagrange multipliers associated with the element boundaries $i$ and $j$, defined at $x=0$ and $x=L_{e}$, respectively, as follows

$\lambda=\left[\begin{array}{ll}\lambda^{i} & \lambda^{j}\end{array}\right]$,

with

$\lambda^{i}=\left[\begin{array}{llll}\lambda_{i}^{N_{1}} & \lambda_{i}^{N_{2}} & \lambda_{i}^{V} & \lambda_{i}^{Q_{s}}\end{array}\right]$

Fig. 3. Analyzed cross-section. 


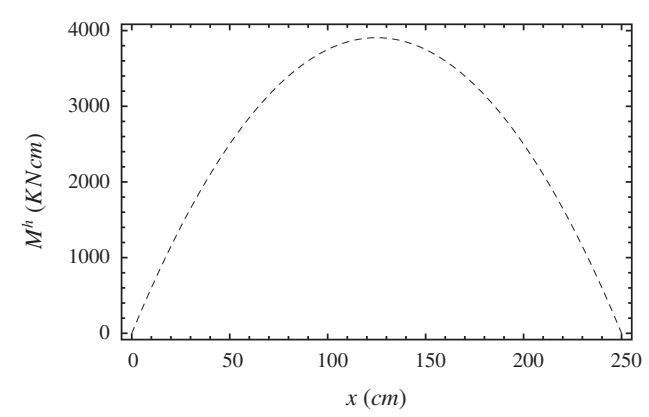

(a)

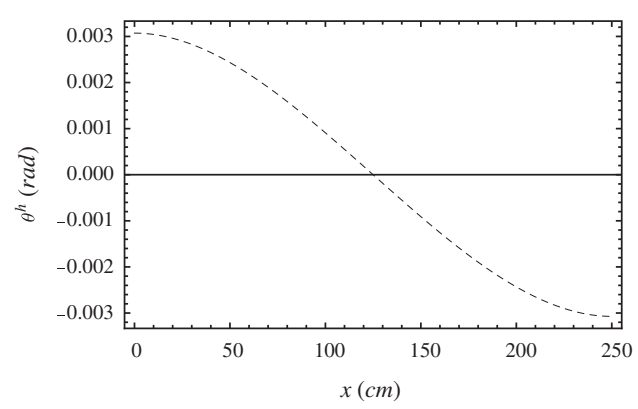

(c)

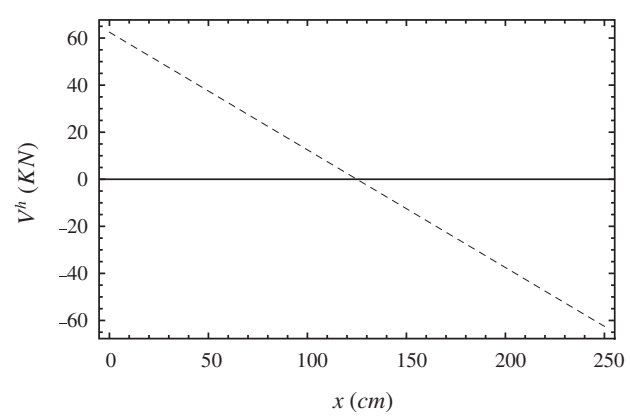

(b)

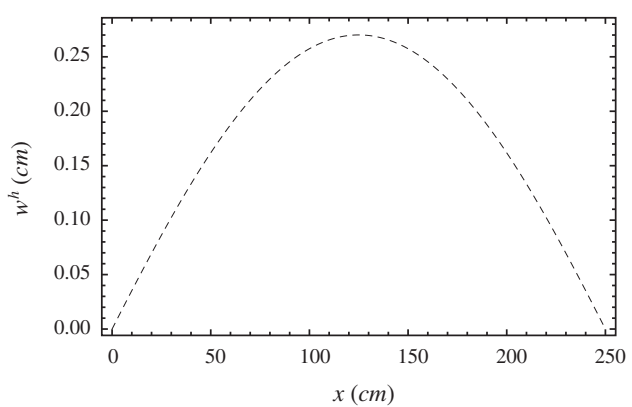

(d)

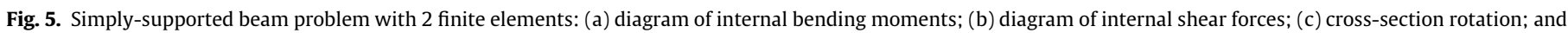
(d) transverse displacement. $k_{s}=0.243 \mathrm{kN} / \mathrm{cm}^{2}$.

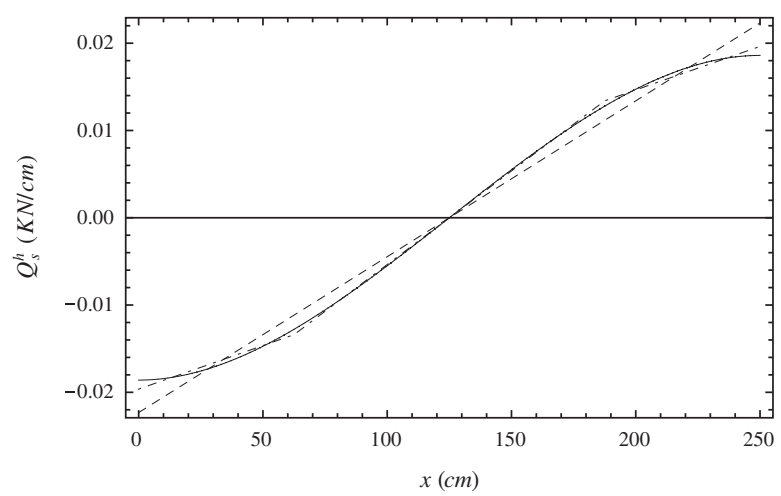

(a)

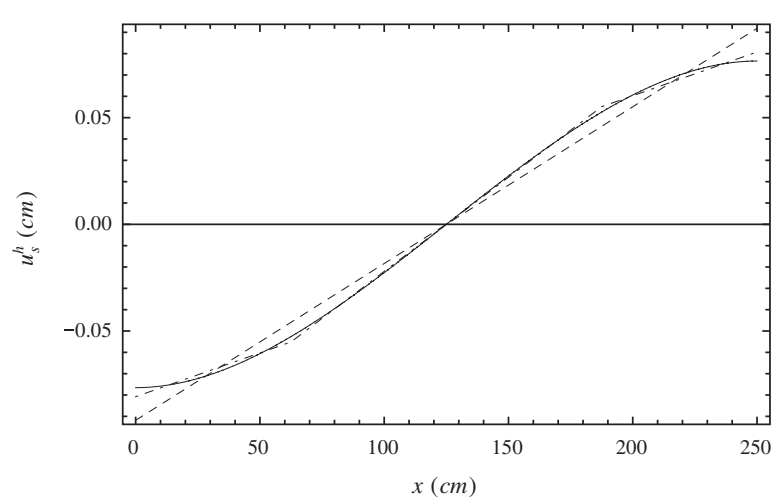

(b)

Fig. 6. Simply-supported beam problem with 2 finite elements (dashed line), 4 finite elements (dot-dashed line) and 128 finite elements (continuous line): (a) interlayer shear flux and (b) interlayer slip displacement. $k_{s}=0.243 \mathrm{kN} / \mathrm{cm}^{2}$. and

$$
\lambda^{j}=\left[\begin{array}{llll}
\lambda_{j}^{N_{1}} & \lambda_{j}^{N_{2}} & \lambda_{j}^{V} & \lambda_{j}^{Q_{s}}
\end{array}\right] .
$$

$\boldsymbol{F}_{e}$ is the element flexibility matrix given as the following decomposition

$\boldsymbol{F}_{e}=\boldsymbol{F}_{e}^{k_{s}}+\boldsymbol{F}_{e}^{I_{1}}+\boldsymbol{F}_{e}^{I_{2}}+\boldsymbol{F}_{e}^{A_{1}}+\boldsymbol{F}_{e}^{A_{2}}+\boldsymbol{F}_{e}^{C}$

with $\boldsymbol{F}_{e}^{k_{s}}, \boldsymbol{F}_{e}^{I_{1}}, \boldsymbol{F}_{e}^{I_{2}}, \boldsymbol{F}_{e}^{A_{1}}, \boldsymbol{F}_{e}^{A_{2}}$ and $\boldsymbol{F}_{e}^{C}$ the flexibility matrices associated with the interlayer slip deformation, bending and axial deformations of beams 1 and 2, and shear deformation of the beam, respectively. $\boldsymbol{A}_{e}$ is the element equilibrium matrix. For the definition of these matrices, the reader is referred to the Appendix.

Simple direct allocation operations on the elementary systems of equations are finally performed to set up the assembled (global) governing system of algebraic equations for the finite element mesh, from which the approximate solutions for all force and bending moment variables as well as the Lagrange multipliers are computed.

The interlayer slip displacement $u_{s}^{h}$ is computed from the constitutive relation (5f). The pseudo-curvature $\kappa^{h}$ can be uniquely obtained either from (5b) or (5c). Once the pseudo-curvature is known, the cross-sectional rotation $\theta^{h}$ is obtained by integration of the compatibility Eq. (3b) along the beam elements. Afterwards, making use of $\theta^{h}$, the transverse displacement $w^{h}$ arises naturally from the integration of (3), where the shear strains $\gamma^{h}$ are obtained directly from the constitutive relation ( $5 a)$. Note that, the constants of integration that arise from the integration of Eqs. (3b) and (3a) along the elements are uniquely determined by enforcing all the interelement compatibility and Dirichlet boundary conditions.

\section{Numerical results}

To validate and assess the accuracy and effectiveness of the proposed hybrid equilibrium element, several benchmark problems 


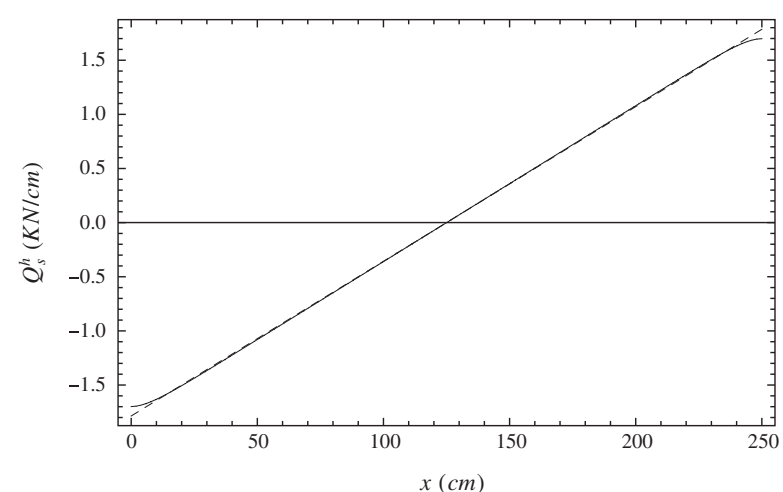

(a)

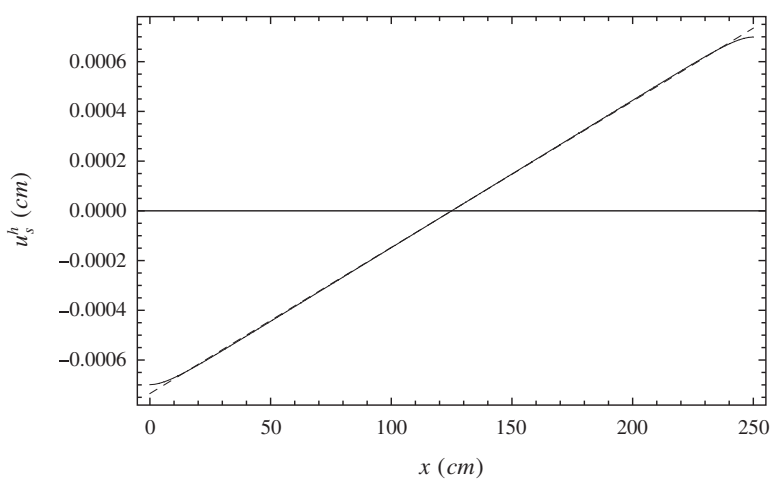

(b)

Fig. 7. Simply-supported beam problem with 2 finite elements (dashed line) and 128 finite elements (continuous line): (a) interlayer shear flux and (b) interlayer slip displacement. $k_{s}=2430 \mathrm{kN} / \mathrm{cm}^{2}$.

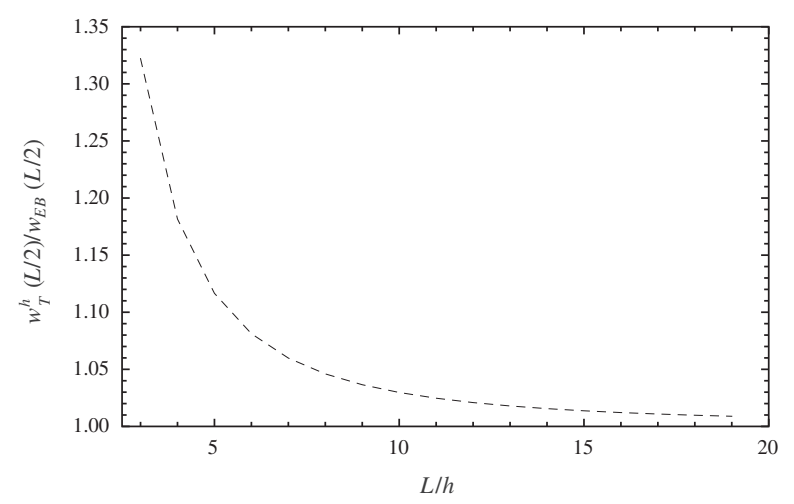

Fig. 8. Simply-supported beam.

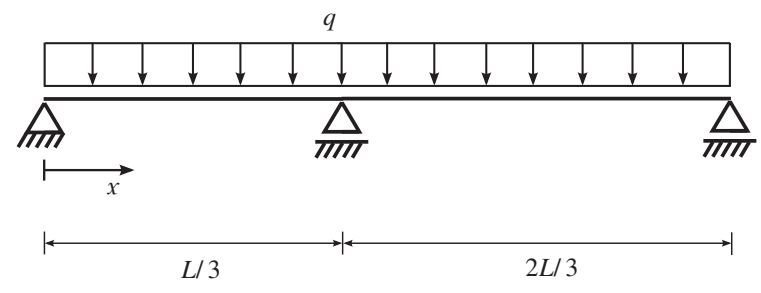

Fig. 9. Continuous beam. are analyzed. The obtained results are compared with the exact analytical solutions when they exit; the latter were computed using the results presented in $[42,16,41]$. The cross-section adopted in all cases is depicted in Fig. 3, with $H_{1}=20 \mathrm{~cm}$, $H_{2}=30 \mathrm{~cm}$ and $b=30 \mathrm{~cm}$. In all problems, the shear correction coefficients were taken as $k_{1}=k_{2}=5 / 6$.

\subsection{Simply-supported beam}

A simply-supported beam under a uniformly distributed load as depicted in Fig. 4 is first considered. The beam length and distributed transverse load were taken as $L=250 \mathrm{~cm}$ and $q=0.5 \mathrm{kN} / \mathrm{cm}$. The material parameters of the beam were taken as $E_{1}=E_{2}=1200 \mathrm{kN} / \mathrm{cm}^{2}, G_{1}=80 \mathrm{kN} / \mathrm{cm}^{2}$ and $G_{2}=120 \mathrm{kN} / \mathrm{cm}^{2}$. As for the interlayer slip modulus, two different values were selected: one corresponding to a connection with very low stiffness, $k_{s}=0.243 \mathrm{kN} / \mathrm{cm}^{2}$, and the other one corresponding to a connection with very high stiffness, $k_{s}=2430 \mathrm{kN} / \mathrm{cm}^{2}$. This problem was first analyzed in [36].

Uniform meshes of 2, 4, 8, 16, 32, 64 and 128 finite elements were considered.

It is of particular interest to study the convergence of the midspan transverse displacement $w^{h}(L / 2)$, mid-span rotation $\theta^{h}(L / 2)$ and interlayer slip $u_{s}^{h}(0)$ of the beam. The results obtained assuming the low stiffness connection are displayed in Table 1. Clearly, the computed results for all quantities converge to their corresponding exact solutions. Most notably, the rotation $\theta^{h}(0)$ obtained on the coarsest mesh already matches the exact one when considering 8 significant digits. As for the mid-span transverse displacement, a mesh of 32 finite elements is required for the numerical solution to match the exact one considering the same precision.

Still assuming the low stiffness connection, the convergence of the mid-span bending moment $M^{h}(L / 2)$, shear force $V^{h}(0)$ and interlayer shear flux $Q_{s}^{h}(0)$ are now studied. The obtained results are displayed in Table 2 . All quantities converge to the exact ones. Further, as it can be seen, both the computed bending moments and shear forces match the exact ones for all meshes.

The diagrams of internal bending moments and shear forces obtained on the 2 finite element mesh are depicted in Fig. 5(a) and (b), respectively. As expected, piecewise-quadratic and piecewise-linear polynomial distributions were produced by the formulation for bending moments and shear forces, respectively. As it can be seen, either the bending moment or shear force distributions are continuous along the inter-element boundaries. In addition, equilibrium conditions $M^{h}(0)=M^{h}(L)=0$ are satisfied. Notably, as the exact solutions for the bending moment and shear force are quadratic and linear polynomials, respectively, as a result of the uniformity of the applied transverse load, the proposed formulation is capable of producing the exact solutions for $M$ and $V$ even on a 2 finite element mesh.

The computed cross-sectional rotation and transverse displacement fields obtained on the 2 finite element mesh are depicted in Fig. 5(c) and (d), respectively. As it can be observed, the solutions for both $\theta$ and $w$ are continuous along the inter-element boundaries. Also, the transverse displacement field satisfies the compatibility boundary conditions given as $w(0)=w(L)=0$. The results on the mesh with 2 finite elements are already very close to their corresponding exact solutions, as it can be concluded from the analysis of Table 1. It was therefore decided not to include the results obtained on the remaining meshes into the figures, as they would seem coincident with the presented ones.

The interlayer slip flux and interlayer slip displacement fields obtained on the 2, 4 and 128 finite element meshes are represented in Fig. 6. The numerical solutions are clearly convergent, with the errors of the solutions being greater near the beam ends. 


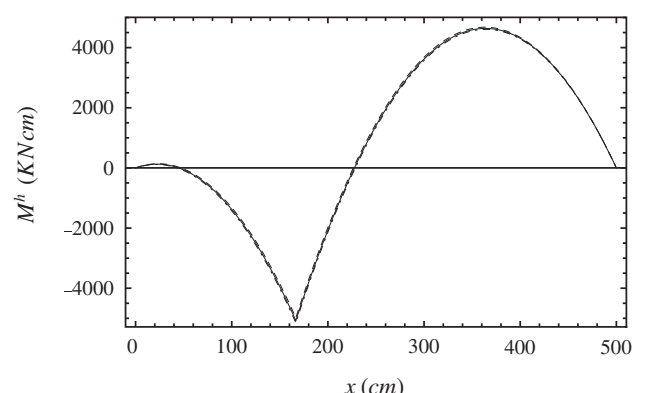

(a)

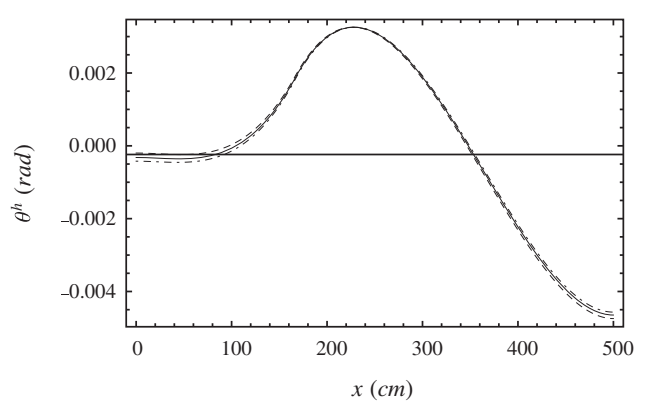

(c)

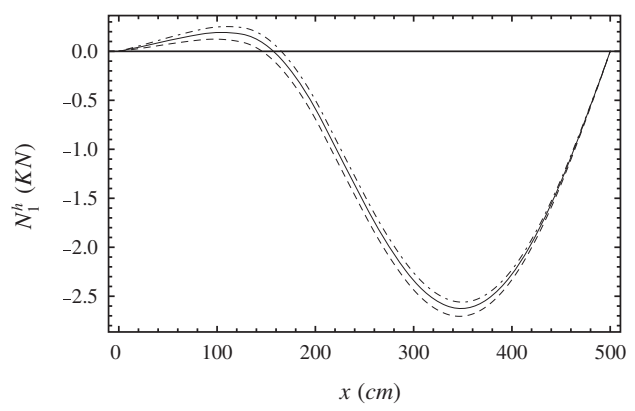

(e)

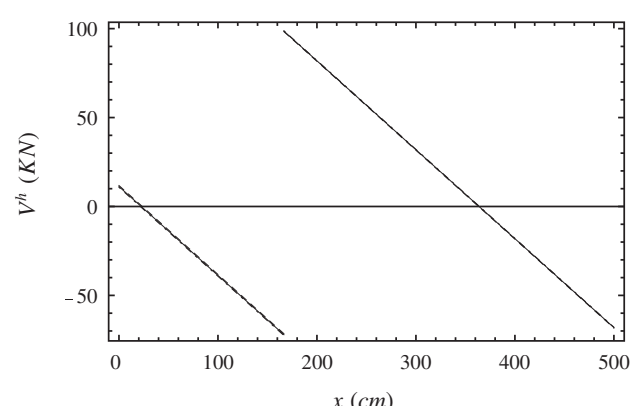

(b)

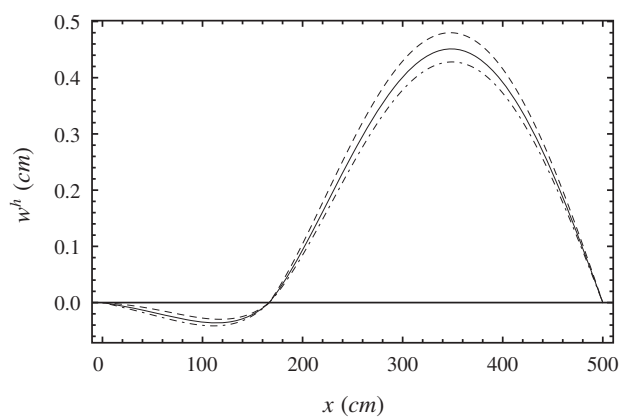

(d)

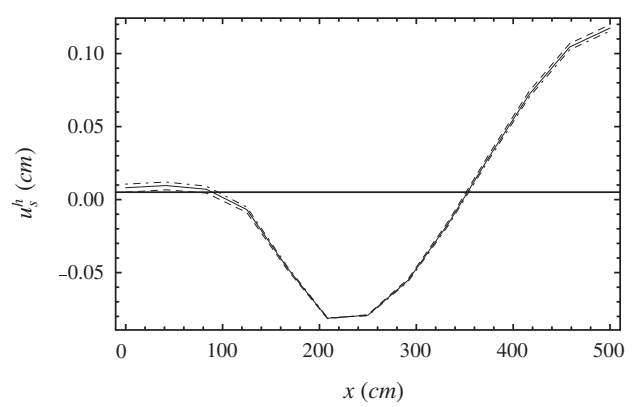

(f)

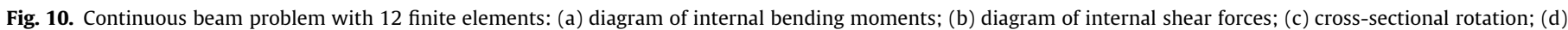
transverse displacement; (e) axial force $N_{1}^{h}$; and (f) interlayer slip displacement. $G=120 \mathrm{kN} / \mathrm{cm}^{2}$ (dashed); $G=240 \mathrm{kN} / \mathrm{cm}^{2}$ (continuous); $G=1200 \mathrm{kN} / \mathrm{cm}^{2}$ (dot-dashed).

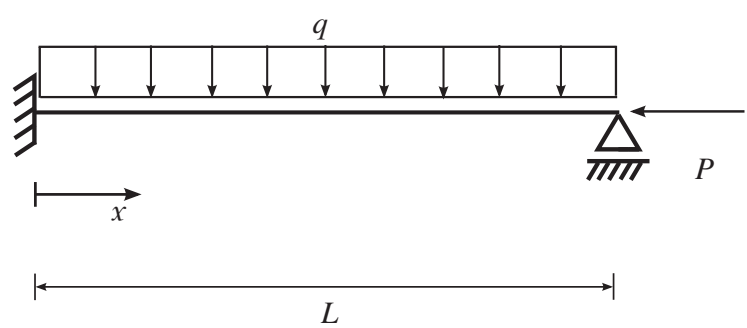

Fig. 11. Clamped-simply-supported beam.

To demonstrate numerically that the proposed finite element formulation is free from slip-locking, the interlayer slip modulus was set to $k_{s}=2430 \mathrm{kN} / \mathrm{cm}^{2}$. The new sets of interlayer shear fluxes and slip displacements obtained on the meshes with 2 and 128 finite elements are depicted in Fig. 7. Clearly, even with the coarsest mesh, the results are already very accurate, being less accurate near the boundaries, and no slip oscillations are observed. This proves the insensitivity of the proposed formulation to the sliplocking phenomenon.
Table 3

Clamped-simply-supported beam problem: mid-span transverse displacement $w^{h}(L / 2)$, interlayer slip $u_{s}^{h}(L)$, and rotation $\theta^{h}(L)$.

\begin{tabular}{rlll}
\hline \multicolumn{1}{r}{$n_{e}$} & $w^{h}(L / 2)(\mathrm{cm})$ & $u_{s}^{h}(L)(\mathrm{cm})$ & $\theta^{h}(L)(\mathrm{rad})$ \\
\hline 2 & 0.13193803 & 0.05282827 & -0.00171587 \\
4 & 0.13190024 & 0.04516104 & -0.00171578 \\
8 & 0.13189884 & 0.04290272 & -0.00171577 \\
16 & 0.13189876 & 0.04227848 & -0.00171577 \\
32 & 0.13189875 & 0.04211478 & -0.00171577 \\
64 & 0.13189875 & 0.04207290 & -0.00171577 \\
128 & 0.13189875 & 0.04206231 & -0.00171577 \\
\hline
\end{tabular}

Finally, the sensitivity of the formulation to shear-locking is numerically assessed. To do so, for different length-to-thickness $L / h$ ratios, the mid-span transverse displacement of the two-layer Timoshenko composite beam obtained with the proposed formulation on a 2 finite element mesh, $w_{T}^{h}(L / 2)$, is compared with the exact value given by the Euler-Bernoulli theory for the same problem, $w_{E B}(L / 2)$, see Fig. 8 . As can be observed, the numerical results for the two-layer Timoshenko beam model converge to the Euler-Bernoulli's solution as the beam becomes thinner. It can 
Table 4

Clamped-simply-supported beam problem: mid-span bending moment $M^{h}(0)$, shear force $V^{h}(0)$, and interlayer shear flux $Q_{s}^{h}(L)$.

\begin{tabular}{rlll}
\hline \multicolumn{1}{c}{$n_{e}$} & $M^{h}(0)(\mathrm{kN} \mathrm{cm})$ & $V_{s}^{h}(0)(\mathrm{kN})$ & $Q_{s}^{h}(L)(\mathrm{kN} / \mathrm{cm})$ \\
\hline 2 & -3719.80059 & 77.4792024 & 0.01283727 \\
4 & -3719.88435 & 77.4795374 & 0.01097413 \\
8 & -3719.88953 & 77.4795581 & 0.01042536 \\
16 & -3719.88985 & 77.4795594 & 0.01027367 \\
32 & -3719.88987 & 77.4795594 & 0.01023389 \\
64 & -3719.88988 & 77.4795595 & 0.01022371 \\
128 & -3719.88988 & 77.4795595 & 0.01022114 \\
\hline
\end{tabular}

therefore be concluded that the proposed finite element is free from shear-locking.

\subsection{Continuous beam}

In order to assess the influence of the shear modulus on the mechanical behaviour of two-layer Timoshenko beam structures [36], a parametric study is now performed for the continuous beam problem depicted in Fig. 9. The material parameters were taken as in the previous problem, i.e., $k_{s}=0.243 \mathrm{kN} / \mathrm{cm}^{2}, E_{1}=E_{2}=1200 \mathrm{kN} / \mathrm{cm}^{2}$, except for the shear modulus, which was assumed to take the following three different values: $G=G_{1}=G_{2}=(120,240,1200) \mathrm{kN} / \mathrm{cm}^{2}$. The length of the beam was taken as $L=500 \mathrm{~cm}$. A uniform transverse distributed load $q=0.5 \mathrm{kN} / \mathrm{cm}$ was considered.

A single uniform mesh of 12 finite elements was considered for the analysis. The left-hand span of the beam was discretized into 4 elements, whereas the right-hand span was discretized into 8 elements.

The solutions obtained for the pairs $(M, V),(\theta, w)$ and $\left(N_{1}, u_{s}\right)$ are depicted in Fig. 10. The first conclusion that can be drawn form the analysis of the figures is that all solutions are continuous across the element boundaries, as well as satisfy the corresponding boundary conditions. In addition, it can be observed that the shear modulus

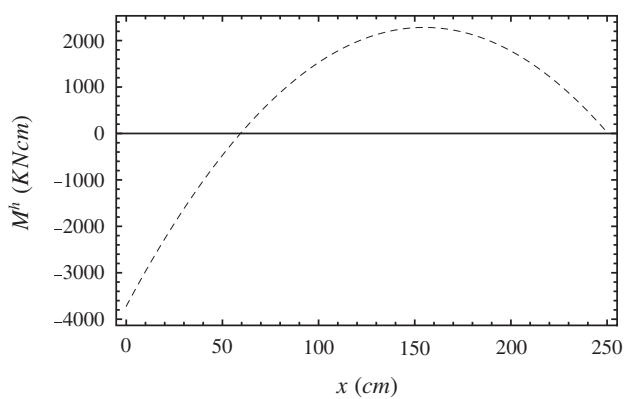

(a)

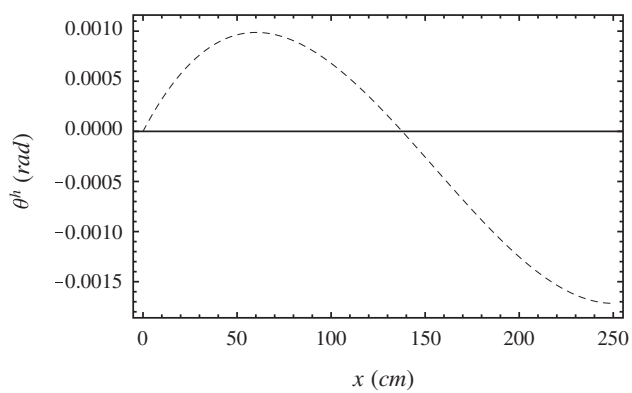

(c) has a strong influence on the structural response of the model. More specifically, while the interlayer slip displacement increases with increasing shear modulus over the left-hand span, it decreases with increasing shear modulus over the right-hand span. The same can be said about the transverse displacement. Also the cross-sectional rotation and axial force fields are affected by the shear modulus. As for the bending moment and shear force fields, the shear modulus has little influence on their distributions.

\subsection{Clamped-simply-supported beam with axial load}

A clamped-simply-supported beam as depicted in Fig. 11 is now considered. The beam is subjected to a uniformly distributed transverse load and an axial force at its simply-supported end. The material parameters were taken as in the simply-supported beam problem, i.e., $k_{s}=0.243 \mathrm{kN} / \mathrm{cm}^{2}, E_{1}=E_{2}=1200 \mathrm{kN} / \mathrm{cm}^{2}, G_{1}=80 \mathrm{kN} /$ $\mathrm{cm}^{2}$ and $G_{1}=120 \mathrm{kN} / \mathrm{cm}^{2}$. The length of the beam was taken as $L=250 \mathrm{~cm}$. The transverse load was set to $q=0.5 \mathrm{kN} / \mathrm{cm}$. The axial load was set to $P=1 \mathrm{kN}$ and assumed to be applied at the centroid of beam 1 .

The analysis was carried out on 2, 4, 8, 16, 32, 64 and 128 uniform finite element meshes.

It is of particular interest to study the convergence of the following quantities: (i) mid-span transverse displacement $w^{h}(L / 2)$, (ii) interlayer slip displacement $u_{s}^{h}(L)$, (iii) cross-sectional rotation $\theta^{h}(L)$, (iv) bending moment $M^{h}(0)$, (v) shear force $V_{s}^{h}(0)$, and (vi) interlayer shear flux $Q_{s}^{h}(L)$. The results obtained for the first three quantities are displayed in Table 3, whereas those obtained for the last three quantities are given in Table 4. As it can be seen, all quantities are convergent. Furthermore, the solutions obtained for quantities (i), (iii), (iv) and (v) are very accurate even on the coarsest mesh. As for quantities (ii) and (vi), finer meshes are required for the formulation to produce relatively accurate solutions.

The solutions obtained for the internal bending moment and shear force fields on the 2 finite element mesh are depicted in Fig. 12(a) and (b), respectively. As was observed in the previous

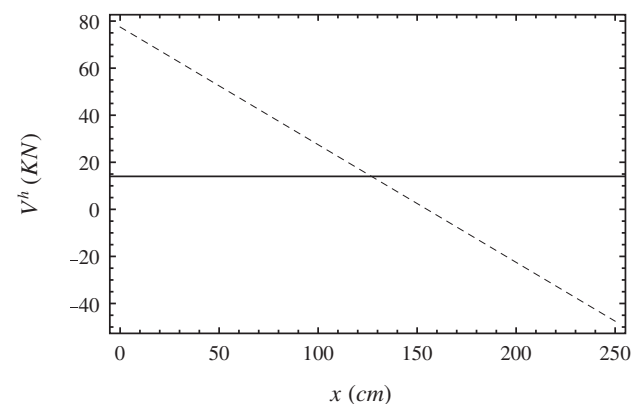

(b)

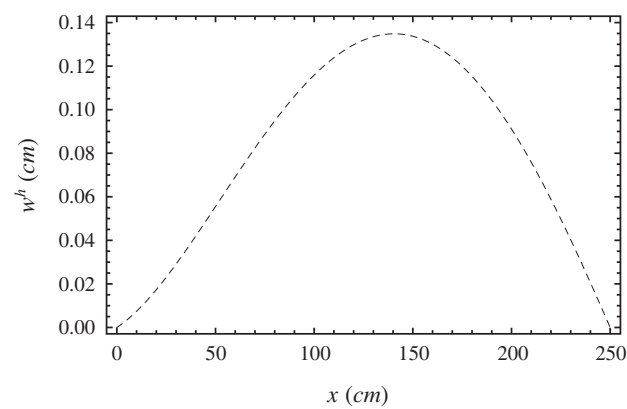

(d)

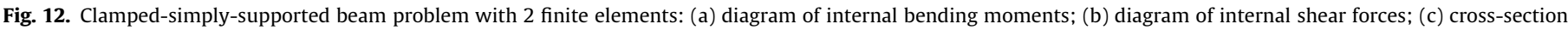
rotation; and (d) transverse displacement. 


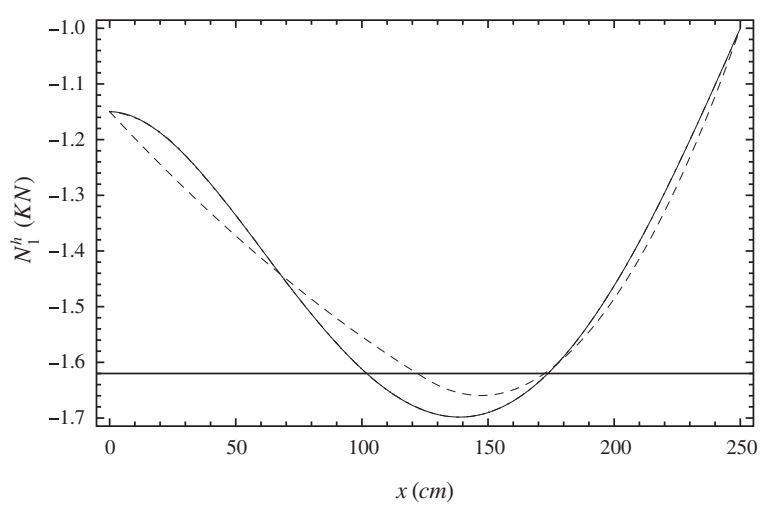

(a)

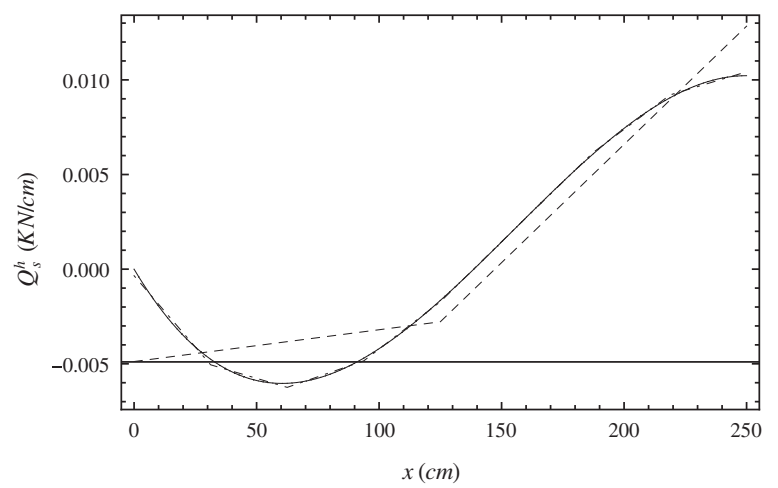

(b)

Fig. 13. Continuous beam problem with 2 (dashed), 8 (dot-dashed) and 128 (continuous) finite elements: (a) axial force on layer 1 and (b) interlayer shear flux.

numerical examples, the obtained distributions are statically admissible. To the best of the authors' knowledge, none of the approaches previously proposed in the literature for layered Timoshenko composite beams with partial interaction have the ability to produce statically admissible stress distributions.

The cross-sectional rotation and transverse displacement fields obtained on the 2 finite element mesh are represented in Fig. 12(c) and (d), respectively. Again, as in the preceding cases, these solutions are continuous and satisfy all the compatibility boundary conditions of the problem, which are defined in this case as $w(0)=w(L)=0$ and $\theta(0)=0$.

The axial force on layer 1 and interlayer shear flux distributions obtained on the 2, 8 and 128 finite element meshes are shown in Fig. 13. As it can be observed, the axial force field satisfies the equilibrium condition $N_{1}(L)=P$. It can also be seen that, while the solutions obtained on the 2 finite element mesh are still relatively inaccurate, the solutions obtained on the 8 finite element mesh almost coincide with those computed on the 128 finite element mesh, which from a numerical point of view, may be regarded as reference solutions.

\section{Conclusions}

A novel finite element formulation for the static analysis of Timoshenko composite beams with partial interaction was introduced. The formulation is variationally consistent with a complementary-energy-based principle only involving force- and moment-like variables as fundamental unknown fields. The approximate fields are chosen so that the force/moment fields are in equilibrium, i.e., internal equilibrium and continuous force/moment transmission between elements are satisfied exactly. The accuracy and effectiveness of the new formulation was assessed numerically through the analysis of several problems. Unlike traditional displacement-based finite element formulations, the proposed formulation is naturally free from both shear- and slip-locking phenomena. The formulation can be extended to both dynamic and buckling scenarios. Another appealing future development is the extension of the proposed formulation to the case of multi-layered beams.

\section{Acknowledgements}

The authors are grateful for the partial financial support provided by FP7-People-2011-IAPP European Union Grant No. 284544.

\section{Appendix A}

The element flexibility matrix is decomposed as

$$
\boldsymbol{F}_{e}^{k_{s}}=\frac{1}{h^{2} k_{s} L}\left[\begin{array}{ccccccccc}
1 & -1 & 0 & 1 & -1 & 0 & 0 & 0 & L \\
-1 & 1 & 0 & -1 & 1 & 0 & 0 & 0 & -L \\
0 & 0 & \frac{16}{3} & 0 & 0 & \frac{16}{3} & 0 & 0 & 0 \\
1 & -1 & 0 & 1 & -1 & 0 & 0 & 0 & L \\
-1 & 1 & 0 & -1 & 1 & 0 & 0 & 0 & -L \\
0 & 0 & \frac{16}{3} & 0 & 0 & \frac{16}{3} & 0 & 0 & 0 \\
0 & 0 & 0 & 0 & 0 & 0 & 0 & 0 & 0 \\
0 & 0 & 0 & 0 & 0 & 0 & 0 & 0 & 0 \\
L & -L & 0 & L & -L & 0 & 0 & 0 & L^{2}
\end{array}\right]
$$

$$
\boldsymbol{F}_{e}^{I_{1}}=\frac{L}{3 E_{1} I_{1}}\left[\begin{array}{ccccccccc}
1 & \frac{1}{2} & 1 & 0 & 0 & 0 & 0 & 0 & 0 \\
\frac{1}{2} & 1 & 1 & 0 & 0 & 0 & 0 & 0 & 0 \\
1 & 1 & \frac{8}{5} & 0 & 0 & 0 & 0 & 0 & 0 \\
0 & 0 & 0 & 0 & 0 & 0 & 0 & 0 & 0 \\
0 & 0 & 0 & 0 & 0 & 0 & 0 & 0 & 0 \\
0 & 0 & 0 & 0 & 0 & 0 & 0 & 0 & 0 \\
0 & 0 & 0 & 0 & 0 & 0 & 0 & 0 & 0 \\
0 & 0 & 0 & 0 & 0 & 0 & 0 & 0 & 0 \\
0 & 0 & 0 & 0 & 0 & 0 & 0 & 0 & 0
\end{array}\right]
$$

$$
\boldsymbol{F}_{e}^{I_{2}}=\frac{L}{3 E_{2} I_{2}}\left[\begin{array}{ccccccccc}
0 & 0 & 0 & 0 & 0 & 0 & 0 & 0 & 0 \\
0 & 0 & 0 & 0 & 0 & 0 & 0 & 0 & 0 \\
0 & 0 & 0 & 0 & 0 & 0 & 0 & 0 & 0 \\
0 & 0 & 0 & 1 & \frac{1}{2} & 1 & 0 & 0 & 0 \\
0 & 0 & 0 & \frac{1}{2} & 1 & 1 & 0 & 0 & 0 \\
0 & 0 & 0 & 1 & 1 & \frac{8}{5} & 0 & 0 & 0 \\
0 & 0 & 0 & 0 & 0 & 0 & 0 & 0 & 0 \\
0 & 0 & 0 & 0 & 0 & 0 & 0 & 0 & 0 \\
0 & 0 & 0 & 0 & 0 & 0 & 0 & 0 & 0
\end{array}\right]
$$

$$
\boldsymbol{F}_{e}^{A_{1}}=\frac{L}{E_{1} A_{1} h^{2}}\left[\begin{array}{ccccccccc}
\frac{1}{3} & -\frac{1}{3} & -\frac{1}{3} & \frac{1}{3} & -\frac{1}{3} & -\frac{1}{3} & -\frac{h}{2} & 0 & \frac{L}{3} \\
-\frac{1}{3} & \frac{1}{3} & \frac{1}{3} & -\frac{1}{3} & \frac{1}{3} & \frac{1}{3} & \frac{h}{2} & 0 & -\frac{L}{3} \\
-\frac{1}{3} & \frac{1}{3} & \frac{8}{15} & -\frac{1}{3} & \frac{1}{3} & \frac{8}{15} & \frac{2 h}{3} & 0 & -\frac{L}{3} \\
\frac{1}{3} & -\frac{1}{3} & -\frac{1}{3} & \frac{1}{3} & -\frac{1}{3} & -\frac{1}{3} & -\frac{h}{2} & 0 & \frac{L}{3} \\
-\frac{1}{3} & \frac{1}{3} & \frac{1}{3} & -\frac{1}{3} & \frac{1}{3} & \frac{1}{3} & \frac{h}{2} & 0 & -\frac{L}{3} \\
-\frac{1}{3} & \frac{1}{3} & \frac{8}{15} & -\frac{1}{3} & \frac{1}{3} & \frac{8}{15} & \frac{2 h}{3} & 0 & -\frac{L}{3} \\
-\frac{h}{2} & \frac{h}{2} & \frac{2 h}{3} & -\frac{h}{2} & \frac{h}{2} & \frac{2 h}{3} & h^{2} & 0 & -\frac{L h}{2} \\
0 & 0 & 0 & 0 & 0 & 0 & 0 & 0 & 0 \\
\frac{L}{3} & -\frac{L}{3} & -\frac{L}{3} & \frac{L}{3} & -\frac{L}{3} & -\frac{L}{3} & -\frac{L h}{2} & 0 & \frac{L^{2}}{3}
\end{array}\right]
$$




$$
\boldsymbol{F}_{e}^{A_{2}}=\frac{L}{E_{2} A_{2} h^{2}}\left[\begin{array}{ccccccccc}
\frac{1}{3} & -\frac{1}{3} & -\frac{1}{3} & \frac{1}{3} & -\frac{1}{3} & -\frac{1}{3} & 0 & -\frac{h}{2} & \frac{L}{3} \\
-\frac{1}{3} & \frac{1}{3} & \frac{1}{3} & -\frac{1}{3} & \frac{1}{3} & \frac{1}{3} & 0 & \frac{h}{2} & -\frac{L}{3} \\
-\frac{1}{3} & \frac{1}{3} & \frac{8}{15} & -\frac{1}{3} & \frac{1}{3} & \frac{8}{15} & 0 & \frac{2 h}{3} & -\frac{L}{3} \\
\frac{1}{3} & -\frac{1}{3} & -\frac{1}{3} & \frac{1}{3} & -\frac{1}{3} & -\frac{1}{3} & 0 & -\frac{h}{2} & \frac{L}{3} \\
-\frac{1}{3} & \frac{1}{3} & \frac{1}{3} & -\frac{1}{3} & \frac{1}{3} & \frac{1}{3} & 0 & \frac{h}{2} & -\frac{L}{3} \\
-\frac{1}{3} & \frac{1}{3} & \frac{8}{15} & -\frac{1}{3} & \frac{1}{3} & \frac{8}{15} & 0 & \frac{2 h}{3} & -\frac{L}{3} \\
-\frac{h}{2} & \frac{h}{2} & \frac{2 h}{3} & -\frac{h}{2} & \frac{h}{2} & \frac{2 h}{3} & 0 & h^{2} & -\frac{L h}{2} \\
0 & 0 & 0 & 0 & 0 & 0 & 0 & 0 & 0 \\
\frac{L}{3} & -\frac{L}{3} & -\frac{L}{3} & \frac{L}{3} & -\frac{L}{3} & -\frac{L}{3} & 0 & -\frac{L h}{2} & \frac{L^{2}}{3}
\end{array}\right]
$$

$$
\boldsymbol{F}_{e}^{C}=\frac{L}{C}\left[\begin{array}{lllllllll}
0 & 0 & 0 & 0 & 0 & 0 & 0 & 0 & 0 \\
0 & 0 & 0 & 0 & 0 & 0 & 0 & 0 & 0 \\
0 & 0 & 0 & 0 & 0 & 0 & 0 & 0 & 0 \\
0 & 0 & 0 & 0 & 0 & 0 & 0 & 0 & 0 \\
0 & 0 & 0 & 0 & 0 & 0 & 0 & 0 & 0 \\
0 & 0 & 0 & 0 & 0 & 0 & 0 & 0 & 0 \\
0 & 0 & 0 & 0 & 0 & 0 & 0 & 0 & 0 \\
0 & 0 & 0 & 0 & 0 & 0 & 0 & 0 & 0 \\
0 & 0 & 0 & 0 & 0 & 0 & 0 & 0 & 1
\end{array}\right]
$$

The element equilibrium matrix is given by

$$
\boldsymbol{A}_{e}=\left[\begin{array}{ccccccccc}
0 & 0 & 0 & 0 & 0 & 0 & 1 & 0 & 0 \\
0 & 0 & 0 & 0 & 0 & 0 & 0 & 1 & 0 \\
\frac{1}{h} & -\frac{1}{h} & 0 & \frac{1}{h} & -\frac{1}{h} & 0 & -1 & -1 & \frac{L}{h} \\
0 & 0 & 0 & 0 & 0 & 0 & 0 & 0 & 1 \\
0 & 0 & 0 & 0 & 0 & 0 & 0 & 0 & -1 \\
0 & 0 & 0 & 0 & 0 & 0 & 0 & 0 & -1 \\
-\frac{1}{h L} & \frac{1}{h L} & \frac{4}{h L} & -\frac{1}{h L} & \frac{1}{h L} & \frac{4}{h L} & 0 & 0 & -\frac{1}{h} \\
\frac{1}{h L} & -\frac{1}{h L} & \frac{4}{h L} & \frac{1}{h L} & -\frac{1}{h L} & \frac{4}{h L} & 0 & 0 & \frac{1}{h}
\end{array}\right]
$$

\section{References}

[1] Argyris J, Tenek L, Mattsson A. BEC: a 2-node fast converging shear-deformable isotropic and composite beam element based on 6 rigid-body and 6 straining modes. Comput Methods Appl Mech Eng 1998;152:281-336.

[2] Arizumi Y, Yamada S, Kajita T. Elastic-plastic analysis of composite beams with incomplete interaction by finite element method. Comput Struct $1981 ; 14: 462-543$.

[3] Ayoub A. A force-based model for composite steel-concrete beams with partial interaction. J Construct Steel Res 2005;61:387-414.

[4] Ayoub A, Filippou FC. Mixed formulation of nonlinear steel-concrete composite beam elements. J Struct Eng (ASCE) 2000;126(3):371-81.

[5] Chakrabarti A, Sheikh AH, Griffith M, Oehlers DJ. Analysis of composite beams with partial shear interactions using a higher order beam theory. Eng Struct 2012;36:283-91.

[6] Chen WQ, Wu YF, Xu RQ. State space formulation for composite beamcolumns with partial interaction. Compos Sci Technol 2007;67(1112):2500-12.

[7] Dall'Asta A, Zona A. Non-linear analysis of composite beams by a displacement approach. Comput Struct 2002;80:2217-28.

[8] Dall'Asta A, Zona A. Slip locking in finite elements for composite beams with deformable shear connection. Finite Elem Anal Des 2004;40(13-14):1907-30.

[9] Dall'Asta A, Zona A. Three-field mixed formulation for non-linear analysis of composite beams with deformable shear connection. Finite Elem Anal Des 2004:40(4):425-48.

[10] Daniels B, Crisinel M. Composite slab behaviour and strength analysis. Part I: calculation procedure. J Struct Eng (ASCE) 1993;119(1):16-35.

[11] Davalos JF, Kim Y, Barbero EJ. Analysis of laminated beams with a layer-wise constant shear theory. Compos Struct 1994;28:241-53.
[12] Fraeijs de Veubeke B. Upper and lower bounds in matrix structural analysis. In: AGARDograph 72: matrix methods of structural analysis. London: Pergamon Press; 1964.

[13] Fraeijs de Veubeke B. Stress analysis. Displacement and equilibrium models in the finite element method. New York: Wiley; 1965. p. 145-97 [chap.].

[14] Erkmen RE, Bradford MA. Treatment of slip locking for displacement-based finite element analysis of composite beam-columns. Int J Numer Methods Eng 2011;85(7):805-26.

[15] Faella C, Martinelli E, Nigro E. Steel and concrete composite beams with flexible shear connection: "exact" analytical expression of the stiffness matrix and applications. Comput Struct 2002;80:1001-9.

[16] Girhammar UA, Pan D. Exact static analysis of partially composite beams and beam-columns. Int J Mech Sci 2007;49:239-55.

[17] Kasper EP, Taylor RL. A mixed-enhanced strain method. Part I: geometrically linear problems. Comput Struct 2000;75:237-50.

[18] Malkus DS, Hughes TJR. Mixed finite element methods-reduced and selective integration techniques: a unification of concepts. Comput Methods Appl Mech Eng 1978;15(1):63-81.

[19] Martinelli E, Faella C, di Palma G. Shear-flexible steel-concrete composite beams in partial interaction: closed-form "exact" expression of the stiffness matrix. J Eng Mech 2012;138(2):151-63.

[20] Martinelli E, Nguyen QH, Hjiaj M. Dimensionless formulation and comparative study of analytical models for composite beams in partial interaction. Construct Steel Res 2012;75:21-31.

[21] Murakami H. A laminated beam theory with interlayer slip. J Appl Mech 1984:51(9):551-9.

[22] Murthy MVVS, Mahapatra DR, Badarinarayana K, Gopalakrishnan S. A refined higher order finite element for asymmetric composite beams. Compos Struct 2005;67:27-35.

[23] Newmark NM, Siess CP, Viest IM. Tests and analysis of composite beams with incomplete interaction. Proc Soc Exp Stress Anal 1951;9(1):75-92.

[24] Oehlers DJ, Bradford MA. Composite steel and concrete structural members: fundamental behaviour. Oxford: Pergamon Press; 1995.

[25] Pian THH, Sumihara K. Rational approach for assumed stress finite elements. Int J Numer Methods Eng 1984;20:1685-95.

[26] Prathap G, Bhashyam GR. Reduced integration and the shear flexible beam element. Int J Numer Methods Eng 1982;18:195-210.

[27] Ranzi G, Bradford MA. Direct stiffness analysis of a composite beam-column element with partial interaction. Comput Struct 2007;85:1206-14.

[28] Ranzi G, Bradford MA, Uy B. A direct stiffness analysis of a composite beam with partial interaction. Int J Numer Methods Eng 2004;61(5):657-72.

[29] Salari MR, Spacone E. Analysis of steel-concrete composite frames with bondslip. J Struct Eng (ASCE) 2001;127(11):1243-50.

[30] Salari MR, Spacone E, Shing PB, Frangopol DM. Nonlinear analysis of composite beams with deformable shear connectors. J Struct Eng (ASCE) 1998;124(10):1148-58.

31] Santos HAFA. Complementary-energy methods for geometrically non-linea structural models: an overview and recent developments in the analysis of frames. Arch Comput Methods Eng 2011;18(4):405-40.

32] Santos HAFA. Variationally consistent force-based finite element method for the geometrically non-linear analysis of Euler-Bernoulli framed structures. Finite Elem Anal Des 2012;53:24-36.

[33] Santos HAFA, Moitinho de Almeida JP. Equilibrium-based finite element formulation for the geometrically exact analysis of planar framed structures. J Eng Mech 2010;136(12):1474-90.

[34] Santos HAFA, Almeida Paulo CI. On a pure complementary energy principle and a force-based finite element formulation for non-linear elastic cables. Int Non-Linear Mech 2011;46(2):395-406.

[35] Santos HAFA, Pimenta PM, Moitinho de Almeida JP. A hybrid-mixed finite element formulation for the geometrically exact analysis of three-dimensional framed structures. Comput Mech 2011;48(5):591-613.

[36] Schnabl S, Saje M, Turk G, Planinc I. Locking-free two-layer Timoshenko beam element with interlayer slip. Finite Elem Anal Des 2007;43(9):705-14.

[37] Simo JC, Hughes TJR. On the variational foundations of assumed strain methods. J Appl Mech 1986:53:51-4.

[38] Subramanian P. Flexural analysis of symmetric laminated composite beams using $C^{1}$ finite element. Compos Struct 2001;54:121-6.

[39] Wu YF, Oehlers DJ, Griffith MC. Partial-interaction analysis of composite beam/ column members. Mech Struct Mach 2002;30(3):309-32.

[40] Xu R, Wang G. Variational principle of partial-interaction composite beams using Timoshenko's beam theory. Int J Mech Sci 2012;60(1):72-83.

[41] Xu R, Wang G. Bending solutions of the Timoshenko partial-interaction composite beams using Euler-Bernoulli solutions. J Eng Mech 2013.

[42] Xu R, Wu Y. Static, dynamic, and buckling analysis of partial interaction composite members using Timoshenko's beam theory. Int J Mech Sci 2007;49(10):1139-55.

[43] Yuan F-G, Miller RE. A higher order finite element for laminated beams. Compos Struct 1990;14:125-50. 